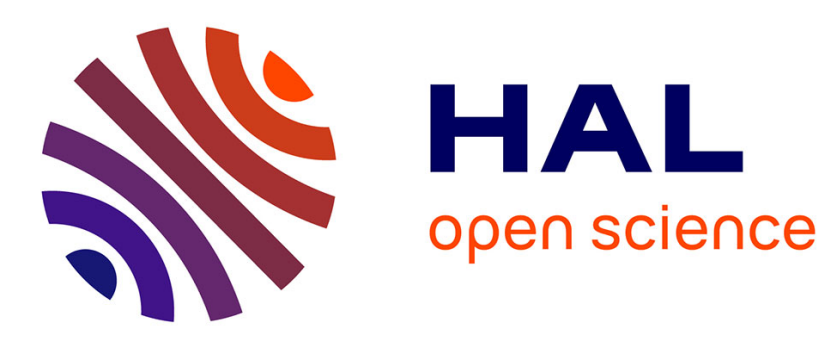

\title{
A Joint Linearization/Companding Approach for Improving a CO-OFDM Transmitter
}

Serban Bejan, Stéphane Azou, Pascal Morel, Cherif Diouf, Mihai Telescu, Noël Tanguy, Ammar Sharaiha

\section{To cite this version:}

Serban Bejan, Stéphane Azou, Pascal Morel, Cherif Diouf, Mihai Telescu, et al.. A Joint Linearization/Companding Approach for Improving a CO-OFDM Transmitter. IEEE Photonics Technology Letters, 2015, 27 (20), pp.2162-2165. 10.1109/LPT.2015.2455674 . hal-01218942

\section{HAL Id: hal-01218942 \\ https://hal.science/hal-01218942}

Submitted on 21 Oct 2015

HAL is a multi-disciplinary open access archive for the deposit and dissemination of scientific research documents, whether they are published or not. The documents may come from teaching and research institutions in France or abroad, or from public or private research centers.
L'archive ouverte pluridisciplinaire HAL, est destinée au dépôt et à la diffusion de documents scientifiques de niveau recherche, publiés ou non, émanant des établissements d'enseignement et de recherche français ou étrangers, des laboratoires publics ou privés. 


\title{
A joint Linearization/Companding Approach for Improving a CO-OFDM Transmitter
}

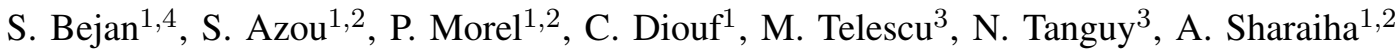

\author{
${ }^{1}$ Ecole Nationale d'Ingénieurs de Brest (ENIB); CNRS, UMR 6285 \\ Lab-STICC; Brest, France; ${ }^{2}$ IRT b<>com, Brest, France \\ ${ }^{3}$ Université de Brest; CNRS, UMR 6285 Lab-STICC; Brest, France \\ ${ }^{4}$ Military Technical Academy, Bucharest, Romania
}

\begin{abstract}
The joint use of peak-to-average power ratio (PAPR) reduction and linearization via digital predistortion is investigated in this letter, with the view to improve the performances of coherent optical OFDM (CO-OFDM) systems employing a semiconductor optical amplifier (SOA). PAPR reduction is performed via Wang's nonlinear companding transform (WNCT), which has been recently pointed out as a pertinent choice for optical communications, and a Filter Lookup Table (FLUT) scheme is considered for linearizing the transmitter. Experimental results prove the effectiveness of the proposed scheme, as a lower EVM is achieved with respect to system implementations using only PAPR reduction or linearization.
\end{abstract}

Index Terms-Coherent Optical OFDM, Semiconductor optical amplifier (SOA), Digital Predistortion, PAPR, Linearization.

\section{INTRODUCTION}

OFDM is considered as a strong candidate for future Wavelength-Division-Multiplexing (WDM) systems due to its many advantages, such as high spectral efficiency, simple compensation of linear channel impairments, dynamic bandwidth allocation capability in a multiuser context, and powerful digital signal processing (DSP)-based implementation [1]. It has been recently demonstrated in [2] that SOA may be a pertinent choice for amplifying Coherent Optical-OFDM $(\mathrm{CO}-\mathrm{OFDM})$, for its interesting features such as large optical bandwidth, small size and possibility of integration at limited cost. Another study [3], conducted in an industrial context, demonstrates the practical feasibility of a SOA-based metro-access infrastructure, with the simultaneous operation of multiple services using different coherent modulation formats. Note also that SOA might be suitable for some low cost coherent systems [4][5]. However, the authors in [2] report that SOA-based systems may suffer from nonlinear effects such as four wave mixing (FWM) and phase-amplitude coupling, as a result of large PAPR that is typically associated with OFDM signals. In a recent paper [6], we considered various companding schemes for improving a SOA-based CO-OFDM system performances. Thanks to an optimization of Wang's predistortion function [7], we obtained a significant Error Vector Magnitude (EVM) reduction and a better power efficiency. This change in the dynamics of OFDM signal translates into less sensitivity to nonlinear channel impairments but the inherent nonlinear behavior of the SOA still significantly affects the performances.
In this letter we investigate the benefits of digital predistortion for linearizing the transmitter characteristics. Such techniques have been widely considered for radio amplifiers [8] but very few results have been reported for optical communication systems so far [9], [10], [11]. An experimental validation of WNCT is addressed here for the first time. Moreover, we propose a joint use of companding and linearization, the latter being performed via a FLUT with a comparison to standard Lookup Table (LUT) implementation; to the knowledge of the authors, such a combination is completely novel.

\section{SYSTEM MODEL}

We first proceeded to the simulation of the CO-OFDM system with the objective of designing and comparing various candidate algorithms for PAPR reduction and linearization. Our implementation relies on a self-developed SOA model [2], which has been fitted to simulate a commercially available bulk $750 \mu \mathrm{m}$ long SOA (INPHENIX-IPSAD1501), so that it yields a very good matching between simulated results and experimental results. The overall structure of the system is illustrated in Fig. 1; it is a classical layout featuring a PAPR reduction block and a predistorter designed to reduce the nonlinear effects occurring between D/A input and SOA output.

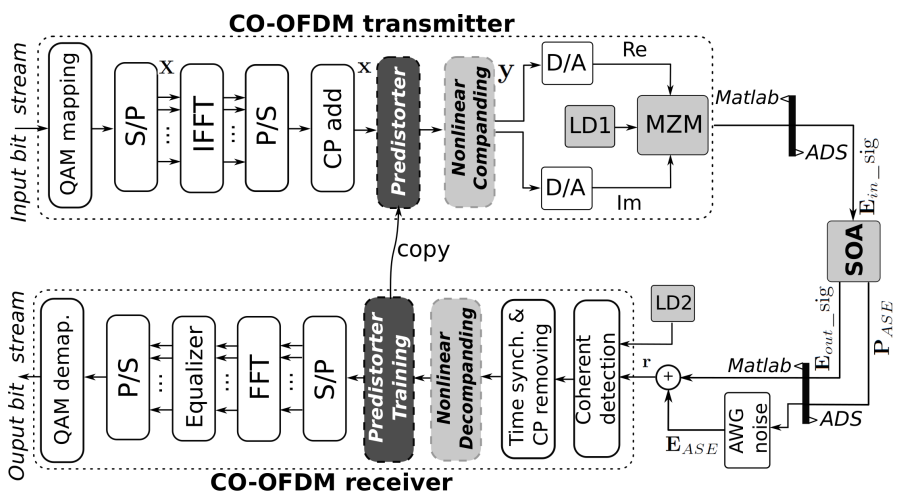

Figure 1. CO-OFDM system with Matlab/ADS co-simulation, including baseband digital predistortion and nonlinear companding blocks (S/P: serial to parallel; IFFT: inverse Fast Fourier Transform; CP: Cyclic Prefix; D/A: Digital to Analog conversion; AWG: Additive White Gaussian).

We recently investigated the issue of PAPR reduction in [6], with a special focus on nonlinear companding techniques. With such methods, the original signal samples $x_{n}$ have their amplitude modified according to a particular nonlinear function $h$, the resulting signal $y_{n}=h\left(x_{n}\right)$ is then converted into analog waveform. At the receiver side, the noisy signal $r_{n}=y_{n}+v_{n}$ is then transformed by the de-companding 
function in order to recover original signal (plus noise): $\widetilde{x}_{n}=h^{-1}\left(r_{n}\right) \simeq x_{n}+h^{-1}\left(v_{n}\right)$. In the present work, we consider that the crest factor reduction is achieved via WNCT [7] as it has proved to be very effective in our previous study (balanced system performances considering criterions such as EVM, complexity and flexibility). Throughout the paper we consider the case of a $5 \mathrm{~Gb} / \mathrm{s}$ QPSK/CO-OFDM transmission spread over 128 subcarriers, with a SOA biased at $200 \mathrm{~mA}$ providing a gain of $19 \mathrm{~dB}$ (with a noise factor of $7 \mathrm{~dB}$ ). The signal is sampled at $24 \mathrm{GS} / \mathrm{s}$ (oversampling factor of 8 ).

\section{LINEARIZATION VIA DIGITAL PREDISTORTION}

\section{A. General principle}

Linearization methods aim at counteracting the nonlinear effects of a device under test (DUT; essentially the SOA in our case) in various ways so that the overall system appears to be linear [8]. A digital baseband predistortion (DPD) is typically adopted as it can compensate irregular characteristics and also efficiently operate over a wide dynamic range. Following this approach, we consider that the DUT is preceded by a DPD block (Fig. 1), denoted as the predistorter, aiming at reproducing the inverse DUT nonlinear behavior. Numerous DPD techniques have been proposed until now, with various performances and complexity. To this day there is no consensus as to the best possible scheme, even though a series of comparisons in terms of performance and complexity have been published, like the one in [12]. Choosing a particular approach is actually closely dependent upon the component to linearize and the application constraints.

LUT is a basic behavioral model for dealing with memoryless nonlinearity (NL). Following this approach a number of correction factors are pre-computed from curve fitting of the amplitude/amplitude (AM/AM) and amplitude/phase (AM/PM) measured characteristics of the DUT. The complex gain associated to the device is then stored in two LUTs, the output of the predistorter being expressed as $x_{\text {out }}(n)=$ $G\left(\left|x_{i n}(n)\right|\right) x_{i n}(n)$ where $G\left(\left|x_{i n}(n)\right|\right)$ is the instantaneous complex gain compensating the nonlinearity and $x_{i n}, x_{\text {out }}$ denoting the input and output signals of the predistorter. This compact implementation may be suitable for real-time applications, but due to quantization errors, the effectiveness is not always guaranteed. Moreover, the LUT scheme exhibits no memory effects, hence it cannot compensate the dynamical nonlinearities of the DUT.

\section{B. The FLUT approach}

In this work, we considered the FLUT scheme initially investigated in [13] which consists in combining a LUT, for the memoryless NL effects, with a codebook of FIR filters (Fig. 2). Hence, the FLUT structure can be considered as an extension of a Hammerstein model in which a static NL is followed by a single linear filter. The LUT is filled with $N_{1}$ complex gain values, a particular gain $G_{i(n)}$ at index $i(n) \in\left\{0,1, \ldots, N_{1}-1\right\}$ being selected according to the input signal magnitude $|z(n)|$, which is uniformly quantized. Hence, the output of the LUT block can be expressed as $z_{l}(n)=G_{i(n)} z(n)$. The index $i(n)$ further determines which filter in the codebook will be applied to the LUT distorted signal $z_{l}(n)$. For a given input magnitude index, the input-output relation characterizing the FLUT system is

$$
z_{l f}(n)=\sum_{k=0}^{L-1} h_{i(n)}(k) G_{i(n-k)} z(n-k)
$$

where $h_{j}$ is the $j$ th filter impulse response of length $L$.

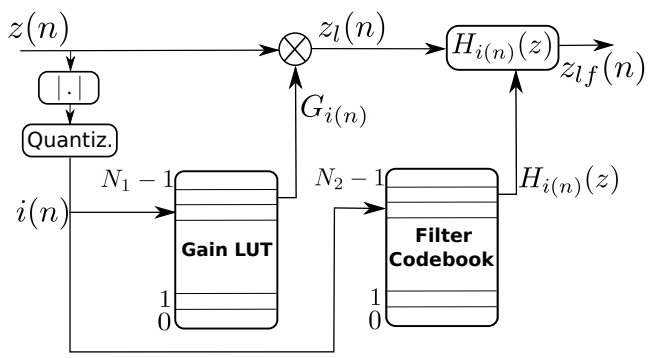

Figure 2. FLUT predistorter $(z(n)$ stands for the baseband OFDM signal)

In the FLUT system, learning is based on the combination of a direct approach for the adaptation of the gains of the LUT and of an indirect approach for the adaptation of the filter impulse responses (see [13] for details). We investigated the linearization via FLUT both in simulations and experimentally. As for every DPD scheme, the key parameters of the FLUT system (namely LUT size $N_{1}$, filter codebook size $N_{2}$, filter length $L$ ) need to be tuned so as to get a good performance improvement. The co-simulation scheme we used, based on an accurate SOA model, largely facilitated this tuning process. Note that the behavior of the linearization block alone is considered in this section, in order to clarify the influence of its key parameters, whereas joint tuning of linearization and PAPR reduction is investigated in the next section (practical experiments). Once the parameters $\left\{N_{1}, N_{2}, L\right\}$ have been chosen, we extracted the FLUT coefficients according to the following three main steps: (1) At the first step, the DPD block is initialized as transparent at the transmitter side. The LUT is loaded with values equal to one, and the impulse responses of the FIR filters are unit impulses. With this initial setting, the output of the predistorter block is equal to its input. At the receiver side the coefficients of the LUT are computed via direct learning (aiming at directly minimizing the error between $z(n)$ and the amplified signal, after some scaling by a reference gain $G_{0}$ ) and the performances of the system without linearization are evaluated; (2) For the second step, on the transmitter side, the LUT has the coefficients previously identified and the filter codebook still has unit impulse responses. On the receiver side, filters impulse responses are computed via indirect learning (in this case a post-distorter is first designed at the DUT output with no predistortion, and then it is used as a predistorter after removing the postdistorter) and a LMS algorithm, and the performance of the system using the LUT block only is evaluated. The adaptation step $\mu$ of the LMS algorithm should be correctly tuned, as it is well known that it can significantly affect the result (in this study we identified $\mu=0.1$ as a pertinent choice). (3) At the third step, the LUT is kept unchanged and the filter codebook is filled with the coefficients determined previously. The performances of FLUT can then be evaluated. Once the FLUT has been identified, it can be used permanently in the 
transmission chain until a major change occurs; then a new learning cycle needs to be performed. It should be emphasized that to ensure a proper operation of the inverse model (the FLUT system here), a one-to-one mapping between the input and the output must be guaranteed. Otherwise, the inversion of the nonlinear effects may not be possible. In case the SOA operates above its saturation threshold, the high peaks of the output signal will be clipped, which may result in inverse computation issues and predistorter divergence. This problem can be partly alleviated by appropriately choosing FLUT parameters, but, as it will subsequently be shown, combining FLUT with a PAPR reduction technique (WNCT here) is instrumental in successfully dealing with it. Another point to be mentioned is that the comparison of the performance of the linearized system with those obtained by the SOA alone should be conducted for the same average output power. In our study, we chose to operate with a reference gain $G_{0}=P_{\max }^{\text {out }} / P_{\max }^{\text {in }}$ with the maximum SOA output power $P_{\max }^{\text {out }}$ being very close to the saturation limit, $P_{\max }^{\text {in }}$ being the corresponding power at SOA input; consequently, the average input power of the linearized system must be larger than that of the SOA alone in order to obtain the same average output power.

The influence of the LUT size $N_{1}$ has been investigated for a codebook of 128 filters of length $L=5$. Two different input power values were considered, $-26 \mathrm{dBm}$ (close to compression point, with an OSNR of $22.5 \mathrm{~dB}$ ) and $-17 \mathrm{dBm}$ (in the saturation region, with an OSNR of $31.9 \mathrm{~dB}$ ). The results (EVM against $N_{1}$ ) are depicted in Fig. 3.a for three different implementations, conventional CO-OFDM system (with no PAPR reduction), linearization via LUT only and linearization via FLUT. For the latter, the cases of a learning sequence and a test sequence are clearly distinguished for a fair evaluation. It can be clearly seen that the FLUT scheme offers better performance with respect to the LUT scheme for low $N_{1}$ values, best results being obtained for 32 bins. Above this threshold some convergence difficulties may occur. Around this optimum LUT size, similar EVM values are noticed.
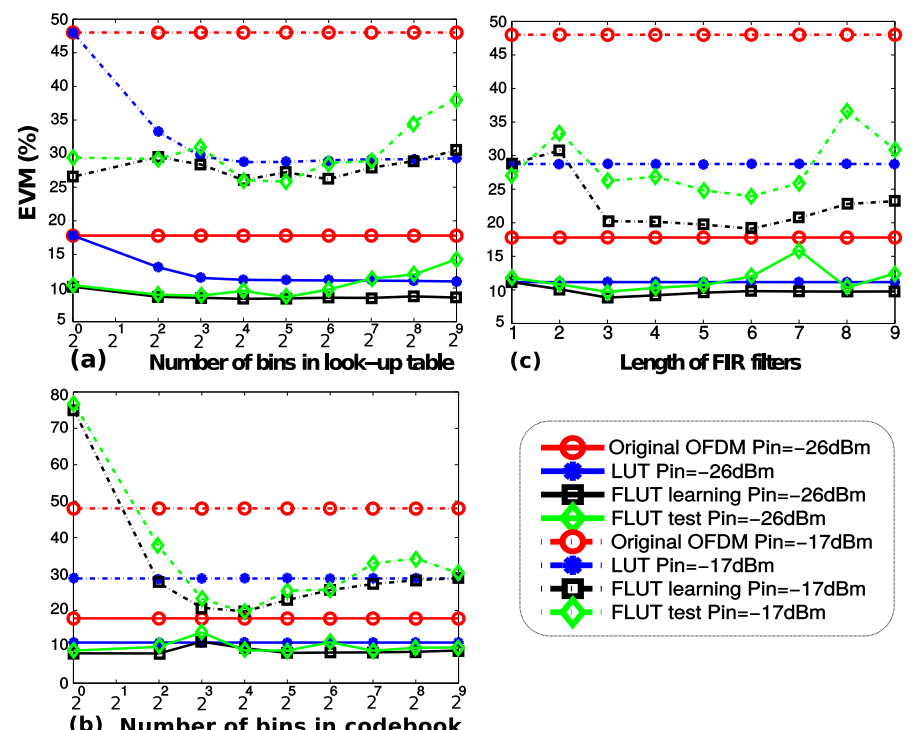

(c)

Length of FIR filters

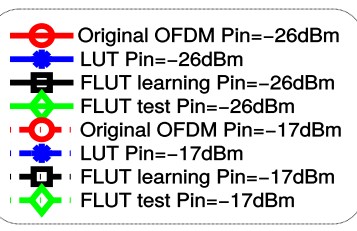

(b) Number of bins in codebook

Figure 3. EVM against various parameters of the FLUT (a) look-up table size (b) codebook size (c) FIR length.
Then, we studied the influence of the filter codebook size $N_{2}$, for $N_{1}=32$ (Fig. 3.b). As can be observed, for $P_{i n}=$ $-17 \mathrm{dBm}$, a local minimum in EVM is reached for $N_{2}=$ 16. For the unsaturated case, any change in $N_{2}$ has almost no impact on EVM, the three implementations giving close performances.

The required length $L$ of the FIR filters is related to the memory effects intrinsic to the SOA. For identifying its value, we evaluated EVM for $L \in[1,9]$. The results are illustrated in Fig. 3.c; a good value of the filter length appears to be $L=3$, for both power values. A slight degradation in performance can be noticed above this value for the saturated case.

We also examined the impact of the signal bandwidth (BW) on the linearization performance (Fig. 4) for two operating points $\left(P_{\text {out }}=-1.3 \mathrm{dBm}, P_{\text {out }}=3.1 \mathrm{dBm}\right)$, by varying the OFDM symbol duration while keeping 128 subcarriers with an oversampling factor of 8 . It can be seen that the SOA behavior remains the same up to $100 \mathrm{MHz}$, FWM being the main nonlinear effect in this range. Between 100 $\mathrm{MHz}$ and $1 \mathrm{GHz}$, the degradation in FLUT performances denotes an increased memory effect, and cross-phase/crossgain modulations (XPM/XGM) tend to appear. A BW above $1 \mathrm{GHz}$ leads to lower amplitude-phase coupling and FWM effect.

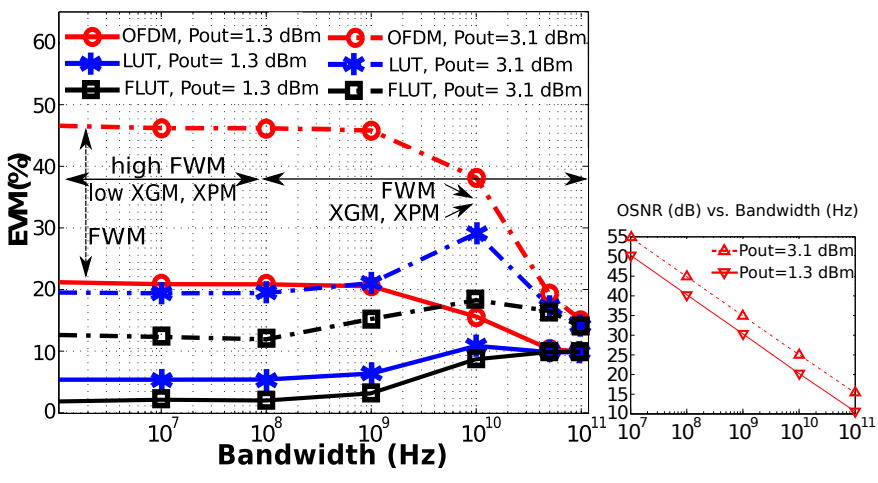

Figure 4. EVM vs. OFDM signal bandwidth for LUT \& FLUT linearizations (left) and OSNR vs. bandwidth (right).

\section{EXPERIMENTAL SETUP AND RESUlts}

Figure 5 shows the experimental setup we considered. As described in the previous section, a preliminary task was the tuning of the FLUT predistorter, together with the WNCT by simulations (Fig. 1). The settings were chosen as follows: $N_{1}=32, N_{2}=16, L=3$ and $\mu=0.1$ for FLUT; $c=0.43$ and $k_{2}=-0.25$ for WNCT. For the WNCT/FLUT combining, the setting was changed by taking $N_{2}=512$ and $L=2$ (these values being identified in simulations in the same way as in section 3), the other coefficients remaining unchanged. The test sequence was a QPSK/OFDM signal with 128 subcarriers, $3 \mathrm{GHz}$ bandwidth, oversampled by a factor of 4 , with a data rate of $5 \mathrm{~Gb} / \mathrm{s}$. Due to memory limit, the total number of OFDM symbols transmitted for one run was limited to 19 (the chosen bandwidth also results from the same constraint), the first symbol being used as a preamble for acquisition; 11 subcarriers were set to zero to establish a guard band on the spectrum sides and 4 subcarriers near DC component were null subcarriers. Moreover, 8 subcarriers were used as pilots, 
the remaining 104 subcarriers being used to transmit data. The signals were generated in Matlab and uploaded to an Agilent M8190A AWG operating at $12 \mathrm{GSa} / \mathrm{s}$; the I/Q components then passed through two RF amplifiers with $16 \mathrm{GHz}$ bandwidth before entering the optical IQ modulator, with a half-wave voltage of about $5.1 \mathrm{~V}$ and a $1540 \mathrm{~nm} \mathrm{CW}$ light applied at its input (ECL laser with $100 \mathrm{kHz}$ linewidth). The transmission link includes only one SOA as a booster. At the receiver side, an Agilent N4391A Optical Modulation Analyzer (OMA) was used as a local oscillator for coherent detection. In order to validate the predistortion techniques, raw signal was recorded at $40 \mathrm{GSa} / \mathrm{s}$ and sent to a receiver implemented under Matlab; the main steps prior to FLUT learning and decompanding steps were timing recovery, phase correction and equalization.

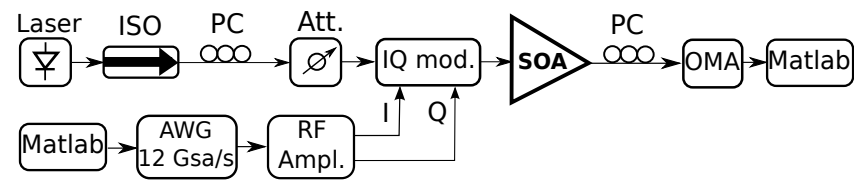

Figure 5. Experimental setup. PC: Polarization controller; Att: Attenuator; IQ Mod: IQ modulator; AWG: Arbitrary waveform generator; ISO: Isolator;

EVM as a function of SOA output power was analysed for different implementations (WNCT, LUT, FLUT, WNCT/LUT, WNCT/FLUT) in comparison with conventional SOA-based CO-OFDM system not using PAPR reduction nor linearization (Fig. 6). First, we can notice the effectiveness of the WNCT scheme once the SOA enters the saturated regime $\left(P_{\text {out }} \geqslant\right.$ $-1.5 \mathrm{dBm}$ ); we get an improvement of around $2.5 \mathrm{~dB}$ at an EVM of $20 \%$, which confirms the simulation results reported in [6]. Second, we observe a significant decrease in EVM by using a linearization scheme, FLUT being slightly better than LUT; however, at high power, convergence difficulties can degrade the performance. Then, it appears that combining WNCT and linearization leads to interesting results, a lower EVM being obtained over a wide range of $P_{\text {out }}$; in particular, we can notice that the FLUT gives the best results in this case. The gain in performance is also evident in the constellations (Fig. 6.b\&c). We also illustrate in Fig. 7 the AM-AM and AM-PM characteristics for conventional system (at $P_{\text {out }}=$ $0.58 \mathrm{dBm}$ ) and with WNCT/FLUT implementation (at $P_{\text {out }}=$ $0.91 \mathrm{dBm}$ ); a huge improvement can be observed, with less scattered points (reduced memory effects) and better linearity.
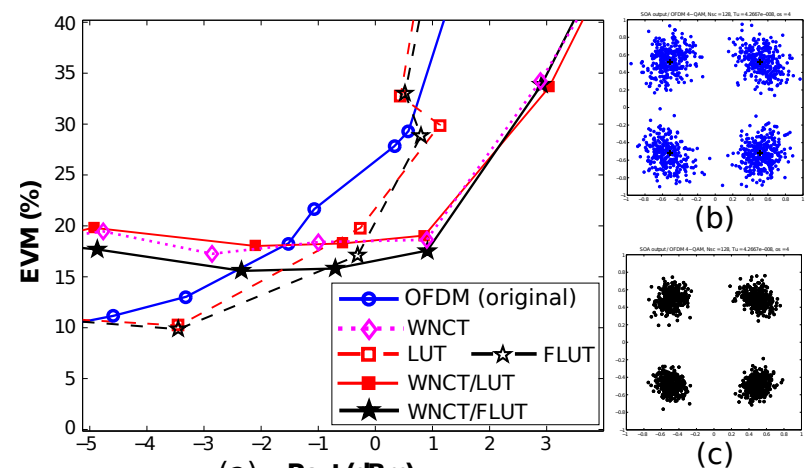

(a) Pout (dBm)

(C)

Figure 6. Experimental peak controlled linearization; (a) EVM vs. output power $\left(P_{\text {out }}\right)(\mathrm{b})$ Constellation for conventional system at $P_{\text {out }}=0.58 \mathrm{dBm}$ (c) Constellation for WNCT/FLUT-based system at $P_{\text {out }}=0.91 \mathrm{dBm}$.
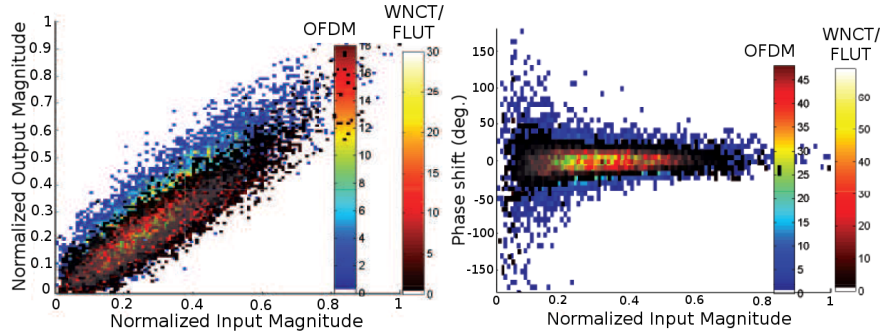

Figure 7. Experimental AM-AM (left) and AM-PM (right) characteristics without and with peak controlled linearization (via WNCT/FLUT).

\section{CONCLUSIONS}

Various predistortion schemes have been investigated for nonlinear effects mitigation in CO-OFDM systems with a special focus on the transmitter part, based on a SOA used as a booster. PAPR reduction is performed via WNCT and a FLUT scheme is considered for linearizing the transmitter. The influence of some key parameters of the FLUT block has been studied by considering a physical model of the SOA. Effectiveness of the WNCT is experimentally proven for the first time and we demonstrate that a joint use of WNCT/FLUT approaches leads to a significant performance improvement in terms of power efficiency and EVM, which can result in a reach extension of the optical link.

\section{REFERENCES}

[1] N. Cvijetic, "OFDM for Next-Generation Optical Access Networks," IEEE J. Lightw. Technol., vol. 30, no. 4, feb. 2012.

[2] H. Khaleghi, P. Morel, A. Sharaiha, and T. Rampone, "Experimental Validation of Numerical Simulations and Performance Analysis of a Coherent Optical-OFDM Transmission System Employing a Semiconductor Optical Amplifier," IEEE J. Lightwave Technol., vol. 31, no. 1, pp. 161-170, January 2013.

[3] H. Schmuck et al., "Demonstration of an SOA-assisted open metroaccess infrastructure for heterogeneous services", Optics Express, vol. 22, Issue 1, 2014.

[4] A. Agmon et al ., "Bi-directional ultra-dense polarization-diverse OFDM/WDM PON with laserless colorless $1 \mathrm{~Gb} / \mathrm{s}$ ONUs based on $\mathrm{Si}$ PICs and <417 MHz mixed-signal ICs," in Proc. OFC 2013 , paper OTh3A.6, 2013.

[5] P. Dong, X. Liu, S. Chandrasekhar, L. L. Buhl, R. Aroca, and Y.$\mathrm{K}$. Chen, "Monolithic silicon photonic integrated circuits for compact $100+\mathrm{Gb} / \mathrm{s}$ coherent optical receivers and transmitters," IEEE J. Sel. Top. Quantum Electron., 20 (4), 2014.

[6] S. Azou, S. Bejan, P. Morel, A. Sharaiha, "Performance Improvement of a SOA-based Coherent Optical-OFDM Transmission System via Nonlinear Companding Transforms", Optics Comm., vol. 336, 2015.

[7] Y. Wang, J. Ge, L. Wang, J. Li, B. Ai, "Nonlinear Companding Transform for Reduction of Peak-to-Average Power Ratio in OFDM Systems," IEEE Trans. Broadcast., vol. 59, no. 2, june 2013.

[8] L. Guan, A. Zhu, "Green Communications: Digital Predistortion for Wideband RF Power Amplifiers", IEEE Microw. Mag., nov./dec. 2014.

[9] L. C. Vieira, N. J. Gomes, A. Nkansah, F. van Dijk, "Behavioral Modeling of Radio-over-Fiber Links Using Memory Polynomials", in Proc. IEEE Int. Topical Meeting on Microw. Photon., pp. 85-88, 2010.

[10] Z. Liu, M. A. Violas, and N. Borges Carvalho, "Digital predistortion for RSOAs as external modulators in radio over fiber systems", Optics Express, vol. 19, no. 18, aug. 2011.

[11] A. Hekkala et al., "Predistortion of Radio Over Fiber Links: Algorithms, Implementation, and Measurements", IEEE Trans. Circ. \& Syst. I, vol. 59, no. 3, march 2012.

[12] A. S. Tehrani, H. Cao, S. Afsardoost, T. Eriksson, M. Isaksson, C. Fager, "A comparative analysis of the complexity/accuracy tradeoff in power amplifier behavioral models", IEEE Trans. Microw. Theory Tech., vol. 58, no. 6, 2010.

[13] P. Jardin, G. Baudoin, "Filter look up table method for power amplifiers linearization”, IEEE Trans. Veh. Technol., vol. 56, pp. 1076-1087, 2007. 